\title{
Análisis multitemporal del cambio en la cobertura del suelo en la Mixteca Alta Oaxaqueña
}

\section{Multitemporal analysis of the change in land cover in the Mixteca Alta Oaxaqueña}

\author{
Rufino Sandoval-García ${ }^{1}$, Rigoberto González Cubas ${ }^{2 *}$ y Javier Jiménez Pérez ${ }^{1}$
}

\begin{abstract}
The aim of this research was to assess the land use and vegetation change in a micro-basin of the Mixteca Alta in the state of Oaxaca, Mexico, from 1995 to 2016, through high resolution images and, thereby, identify the dynamics of loss and gain of the vegetal cover that allow to classify priority areas for conservation and restoration. The analysis was developed through an unsupervised classification and field validation. Results showed that forest cover has a positive increase of $6.6 \%$ during the period from 1995 to 2016, as an outcome of restoration strategies promoted by indigenous communities and government institutions; agriculture decreased by $8.4 \%$, which is largely due to the fact that many areas have been abandoned due to migration and loss of soil productivity, as well as alteration of the precipitation regime, thus presenting a natural conversion to grasslands. The areas without vegetation decreased by $4.1 \%$ as a result of the efforts in the construction of works for soil conservation, formation of successive terraces and stabilization of slopes. The increase in grassland areas within the micro-basin is a cause for concern, since should be considered as priority restoration areas due to the easily erodible soil type.
\end{abstract}

Key words: Land cover, indigenous communities, functional connectivity, desertification, satellite images, micro-watershed.

\section{Resumen}

El objetivo de la presente investigación fue evaluar el cambio del uso de suelo y vegetación en una microcuenca de la Mixteca Alta Oaxaqueña durante el periodo de 1995 a 2016, mediante el uso de imágenes de alta resolución y con ello identificar la dinámica de pérdida y ganancia de la cobertura vegetal que permitan catalogar zonas prioritarias para la conservación y restauración. El análisis se desarrolló con una clasificación no supervisada y validación en campo. Los resultados mostraron un incremento positivo de $6.6 \%$ durante el período de 1995 al 2016 en la cobertura forestal, a partir de las estrategias de restauración impulsadas por las comunidades indígenas e instituciones gubernamentales; mientras que, la agricultura tuvo una disminución de $8.4 \%$, lo cual se explica, en gran medida, porque muchas áreas han sido abandonadas por la migración y la pérdida de productividad del suelo, así como a la alteración del régimen de precipitación; lo que condujo a una conversión natural hacia los pastizales. Las áreas sin vegetación disminuyeron $4.1 \%$ a consecuencia de los esfuerzos en la construcción de obras para la conservación de suelos, la formación de terrazas sucesivas y la estabilización de taludes. El aumento de áreas de pastizal dentro de la microcuenca es un motivo de preocupación, ya que se deben considerar como áreas prioritarias de restauración debido al tipo de suelo fácilmente erosionable.

Palabras clave: Cobertura del suelo, comunidades indígenas, conectividad funcional, desertificación, imágenes satelitales, microcuenca.

Fecha de recepción/Reception date: 22de julio de 2020

Fecha de aceptación/Acceptance date: 20 de enero de 2021

${ }^{1}$ Universidad Autónoma de Nuevo León. Facultad de Ciencias Forestales. México.

${ }^{2}$ Tecnológico Nacional de México / Instituto Tecnológico Superior de San Miguel El Grande. México.

*Autor para correspondencia; correo-e: rigocubas_11@hotmail.com 


\section{Introducción}

La Mixteca Alta Oaxaqueña está catalogada como una zona de desastre ecológico, debido a sus altos índices de erosión que están presentes en más de $60 \%$ de su

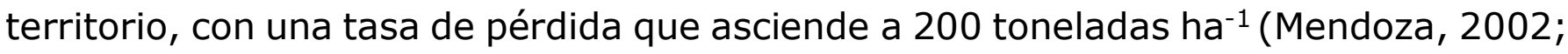
Guerrero-Arenas et al., 2010); principalmente, por el abandono de la agricultura, el sobrepastoreo y el uso de la agricultura tradicional; situación que provocó una alta vulnerabilidad, favorecida por las pendientes pronunciadas, precipitación y suelos arcillosos fácilmente erosionables (Caballero, 2006; Santiago-Mejía et al., 2018).

Las comunidades son vulnerables a los procesos de cambios de uso de suelo, razón por la cual se han realizado diversos estudios relacionados con la degradación, la erosión y la restauración (Contreras-Hinojosa et al., 2003; Valencia et al., 2006), así como de los problemas sociales que enfrentan (Hernández et al., 2011; AguilarSantelises y del Castillo, 2013). Sin embargo, pocos trabajos se han enfocado en un análisis más detallado del proceso de cambio de la cobertura del suelo a nivel de cuenca o microcuenca, como una solución integral para que las estrategias tengan mayor impacto social, ambiental y económico.

En las investigaciones científicas para determinar los procesos de cambio de uso de suelo se utilizan imágenes satelitales principalmente Landsat y Sentinel (Velázquez et al., 2003; Camacho-Sanabria et al., 2015; Steinhausen et al., 2018), las cuales se consideran de baja resolución, además presentan una serie de limitantes que generan un margen de clasificación errónea entre 10 y 20 \% (Pham et al., 2016; Azzari et al., 2017; Astola et al., 2019); por tal motivo, el objetivo del presente proyecto consistió en evaluar el cambio del uso de suelo y la vegetación en una microcuenca de la Mixteca Alta Oaxaqueña durante el periodo 1995-2016, mediante el uso de imágenes de alta resolución, y con ello identificar la dinámica de pérdida y ganancia de la cobertura vegetal que permitan catalogar zonas prioritarias para la conservación y restauración. 


\section{Materiales y Métodos}

El área de estudio comprende dos microcuencas (San Isidro Peñasco (20-082-01007) y San Sebastián Atoyaquillo (20-0082-01-015), las cuales se unificaron para el presente análisis y para fines de la presente investigación se le denominó microcuenca San Isidro Peñasco. Se ubica entre las coordenadas geográficas: 17011'43.57"-

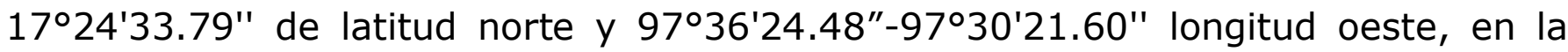
región de la Mixteca Alta del estado de Oaxaca (Inegi, 2004), y tiene una superficie de 16807.58 ha (Figura 1).

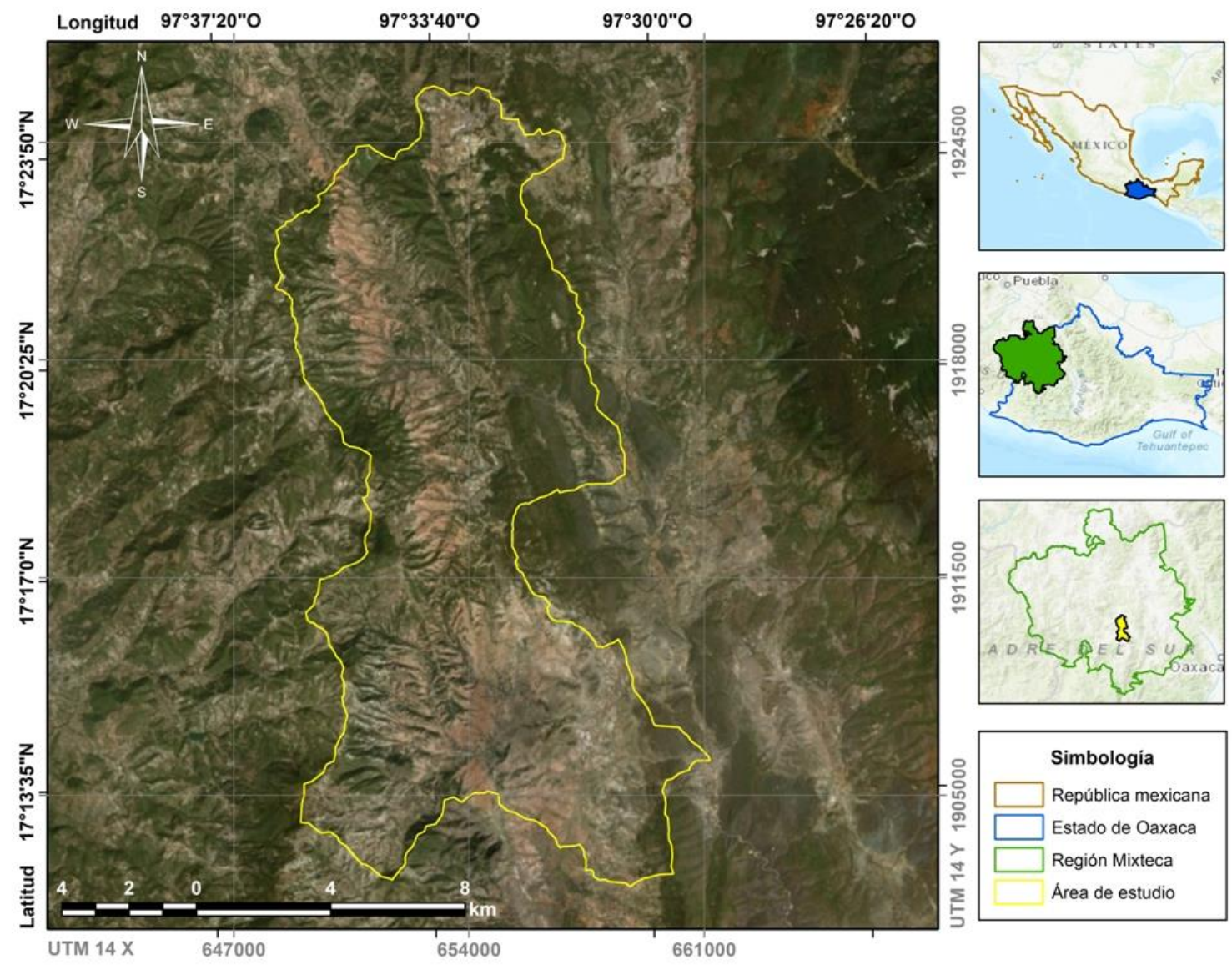

Figura 1. Localización del área de estudio.

El clima dominante es templado subhúmedo $C\left(W_{2}\right), C\left(w_{1}\right)$ y semicálido húmedo $A(C) W_{1}$; los suelos son de tipo Cambisol, Feozem, Leptosol, Luvisol y Regosol (Inegi, 2004). La 
precipitación pluvial varía de $741 \mathrm{~mm}$ a $2200 \mathrm{~mm}$ al año y la temperatura promedio anual es de $27.5^{\circ} \mathrm{C}$ (García-Conabio, 1998; SMN, 2010).

\section{Selección de las imágenes satelitales}

Para un análisis previo del área de estudio, se utilizaron imágenes Landsat 8 OLI (30 m/pixel) y Sentinel 2A (10 m/pixel) del año 2017 (Copernicus Open Access Hub, 2020; USGS, 2020). A las imágenes se les hizo una corrección atmosférica para realizar una clasificación supervisada, y se recortaron geométricamente para ajustar a la extensión del área de interés. Posteriormente, se procesaron y generaron cuatro ortomosaicos compuestos por imágenes de resolución alta: 15 ortofotos del Instituto Nacional de Estadística y Geografía (Inegi) del año 1995 (1.5 m/pixel), 321 imágenes de Birdseye (0.28 m/pixel; 2010), 80 de World Imagery (1.14 m/pixel; 2013) y 80 de Airbus Defence and Space (1.14 m/pixel; 2016).

Las imágenes se obtuvieron de SASPlanet, el cual es un software libre para la visualización y la descarga de ortomosaicos provenientes de los principales proveedores satelitales de manera gratuita y de código abierto como Google Earth, Bing Maps, DigitalGlobe, Kosmosnimki, Yandex Maps, Yahoo! Maps, VirtualEarth, Gurtam, OpenStreetMap, eAtlas, iPhone Maps, General Staff mapsy, entre otros (SASPlanet, 2020).

\section{Clasificación supervisada}

La detección de cambios es el proceso de identificar la diferencia en el estado de un objeto o fenómeno observándolo en diferentes momentos, esencialmente, implica la capacidad de cuantificar efectos temporales mediante conjuntos de datos multitemporales (Lloyd, 1982; Singh, 1989). El uso del suelo y la vegetación se analizó mediante una clasificación no supervisada del módulo $K$-Means Cluster Analysis (KMC), la cual es uno de los métodos de agrupamiento en conglomerados más utilizados para analizar las características en imágenes satelitales (Rashmi et al., 
2016), debido a que es uno de los algoritmos de aprendizaje que resuelven el problema de agrupamiento mediante la técnica de refinamiento iterativo (Oliver et al., 2006; Jumb et al., 2014).

La clasificación se realizó con sitios de entrenamiento de la información obtenida en campo (Figura 2), en la que se ejecutó un total de 10 clasificaciones automatizadas, las cuales se resumieron en siete clases de cobertura vegetal y uso del suelo, con el método de análisis de conglomerados de datos multivariados Distancia mínima iterativa (Hastie et al., 2009). En todos los procedimientos se utilizó el programa de cómputo Qgis 2.18. Las Palmas (QGIS Development Team, 2020), el cual integra la caja de herramientas de procesos SAGA (System for Automated Geoscientific Analyses) de código abierto con licencia de General Public License (Conrad et al., 2015).
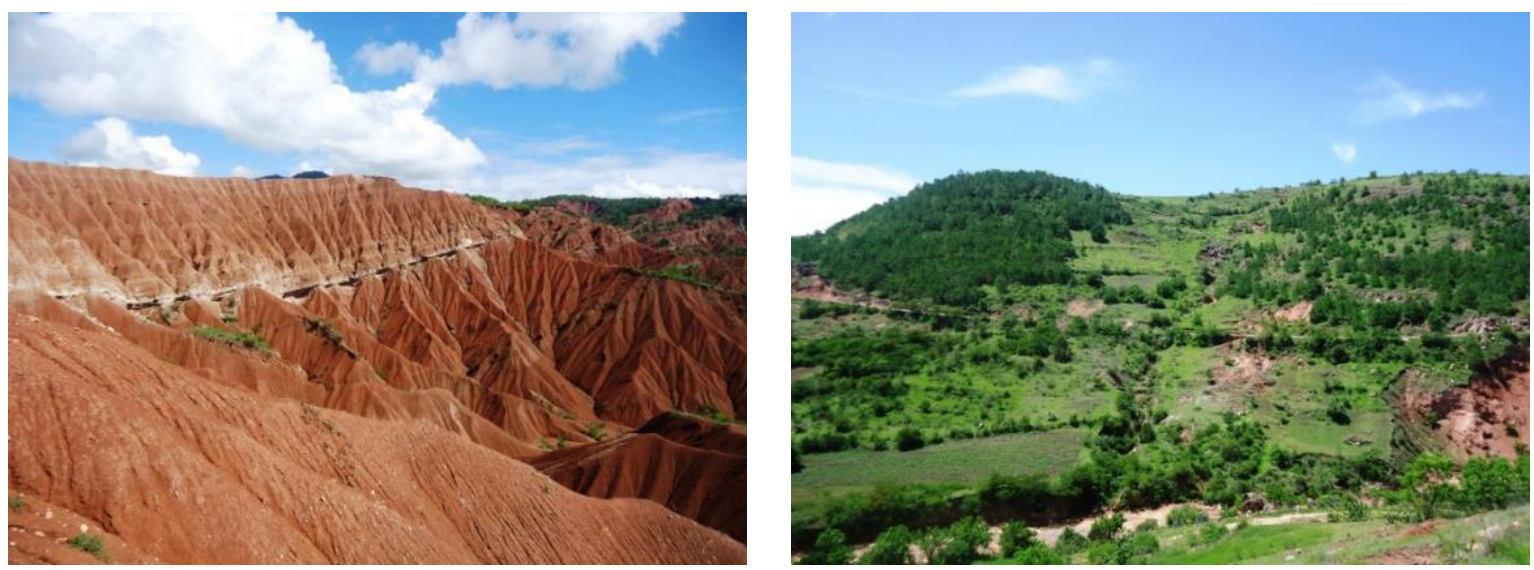

Figura 2. Escenario actual de áreas sin vegetación y de reforestaciones.

\section{Cambio de cobertura vegetal y uso del suelo}

La determinación de la pérdida o ganancia de cobertura de los diferentes usos del suelo y vegetación se obtuvo mediante la construcción de cuatro matrices de transición y una de tasa de cambio para los periodos evaluados. A fin de determinar la tasa de cambio, se usó la ecuación adaptada por Palacio-Prieto et al. (2004): 


$$
\delta_{n}=\left(\left[\frac{S_{2}}{S_{1}}\right]^{l / n}-1\right) * 100
$$

Donde:

$\delta_{n}=$ Tasa de cambio expresada en porcentaje

$S_{1}=$ Superficie de la fecha 1

$S_{2}=$ Superficie de la fecha 2

$n=$ Número de años entre las dos fechas

\section{Descripción del cambio de uso del suelo y vegetación}

Para describir los cambios de cobertura vegetal y uso de suelo, se generó una tabulación cruzada del tiempo 1 y del tiempo 2, la cual permite obtener una matriz de cambios de uso. La diagonal muestra la cantidad total del paisaje estable entre una fecha y otra; mientras que fuera de la diagonal se ubican las transiciones de las clases entre Año 1 y Año 2 . Un valor positivo de la tasa de cambio indica ganancia de superficie, y uno negativo corresponde a una pérdida.

A partir de matrices de transición se calculó la ganancia y pérdida, resultado de la diferencia entre el total del año 2016 y la persistencia. La pérdida se obtuvo de la diferencia entre el total del año 1995 y la persistencia. El intercambio entre categorías se calculó como dos veces el valor mínimo de las ganancias y las pérdidas, y el cambio total para cada categoría se estableció como la suma de las ganancias y las pérdidas. En el caso de las ganancias, se asume que la ganancia por categoría y la proporción de cada categoría del año 2016 son fijas, para después distribuir la ganancia a través de las otras categorías, según la proporción relativa de las otras categorías del año 1995. 


\section{Resultados y Discusión}

\section{Cambios en el uso de suelo y vegetación}

A partir del proceso de clasificación supervisada se derivaron siete clases de uso de suelo y vegetación; sin embargo, las categorías de asentamientos humanos, caminos y ríos no mostraron un cambio significativo, al ocupar $10 \%$ de superficie a lo largo del periodo bajo estudio; con base en esto, solamente se consideraron las siguientes categorías para el análisis: forestal, pastizal, agricultura y áreas sin vegetación.

La microcuenca ocupa una superficie de 15119.02 ha, de la cual $40.8 \%$ correspondió a cubierta forestal y $49.1 \%$ a cubierta no forestal (Cuadro 1 ); estos resultados difieren de los de Carranza (2014) para la región de la Mixteca, pues considera una cubierta forestal de $69 \%$ y áreas no forestales de $31 \%$, lo cual se explica porque utilizaron imágenes satelitales de tipo Spot-5 y Landsat que presentan errores geométricos y nubosidad, así como una resolución que varía de 2.5 a 15 m/pixel para bandas pancromáticas y 10 a $60 \mathrm{~m} /$ pixel para las bandas multiespectrales; mientras que en el presente estudio, la resolución fue de 0.28 y $1.14 \mathrm{~m} / \mathrm{pixel}$ con baja nubosidad.

Cuadro 1. Clasificación del uso del suelo del periodo 1995 al 2016.

\begin{tabular}{|c|c|c|c|c|c|c|c|c|}
\hline \multirow{2}{*}{$\frac{\text { Año }}{\text { Cobertura/Uso }}$} & \multicolumn{2}{|c|}{1995} & \multicolumn{2}{|c|}{2010} & \multicolumn{2}{|c|}{2013} & \multicolumn{2}{|c|}{2016} \\
\hline & ha & $\%$ & ha & $\%$ & ha & $\%$ & $\mathrm{Ha}$ & $\%$ \\
\hline Forestal & 5734.6 & 34.1 & 6804.5 & 40.4 & 6833.9 & 40.6 & 6857.3 & 40.8 \\
\hline Pastizal & 3229.1 & 19.2 & 3518.7 & 20.9 & 3849.6 & 22.9 & 4231.3 & 25.1 \\
\hline Agricultura & 3938.3 & 23.4 & 2918.4 & 17.3 & 2860.4 & 17.0 & 2526.6 & 15.3 \\
\hline Sin vegetación & 2217.3 & 13.1 & 1877.3 & 11.1 & 1575.5 & 9.3 & 1504.0 & 8.9 \\
\hline
\end{tabular}

El uso de suelo y vegetación desde el año 1995 hasta 2016 dentro de la microcuenca fue muy dinámica. Las secuencias comunes observables de los cambios en la cubierta del suelo incluyeron la conversión de agricultura a pastizal, agricultura en áreas sin 
vegetación, agricultura a forestal, pastizal en agricultura, pastizal a forestal, forestal a agricultura y áreas sin vegetación a pastizal (Cuadro 2).

Cuadro 2. Cambios en la cobertura del suelo de 1995 a 2016.

\begin{tabular}{lcccccc}
\hline \multicolumn{1}{c}{ Periodo } & \multicolumn{2}{c}{$\boldsymbol{\Delta 1 9 9 5 - 2 0 1 0}$} & \multicolumn{2}{c}{$\boldsymbol{\Delta 2 0 1 0 - 2 0 1 3}$} & \multicolumn{2}{c}{$\boldsymbol{\Delta 2 0 1 3 - 2 0 1 6}$} \\
\hline Cobertura/Uso & ha & \% & ha & \% $\boldsymbol{H}$ & Ha & \% $\boldsymbol{\Delta}$ \\
\hline Forestal & 1069.8 & 8.5 & 29.4 & 0.2 & 23.4 & 0.1 \\
\hline Pastizal & 289.7 & 4.2 & 330.8 & 4.4 & 381.4 & 4.7 \\
\hline Agricultura & -1019.9 & -14.8 & -57.9 & -1.0 & -333.8 & -6.2 \\
\hline Sin vegetación & -339.6 & -8.0 & -302.3 & -8.7 & -71.0 & -2.3 \\
\hline \multicolumn{5}{c}{$\Delta=$ Incremento }
\end{tabular}

En 1995, la cobertura principal era de tipo forestal (34.1 \%) (Figura 3A), con distribución marcada en la parte alta de la microcuenca; estrategia de conservación del suelo impulsada por las comunidades indígenas, debido a la importancia del papel que desempeña la vegetación en esta zona como área de recarga hídrica y de protección contra la escorrentía superficial y la erosión del suelo (Castellanos-Bolaños y Ruiz, 1993; Champo-Jiménez et al., 2012). La segunda cobertura dominante en este periodo fue la agricultura (23.4\%); como resultado del Sistema Alimentario Mexicano (SAM) en 1980, el Programa Nacional de Alimentos (PNA) en 1983, el programa Lluvia-Tequio-Alimento en 1987 y el Programa Especial para la Seguridad Alimentaria (PESA) en 1994 (Guerrero-Arenas et al., 2010). 

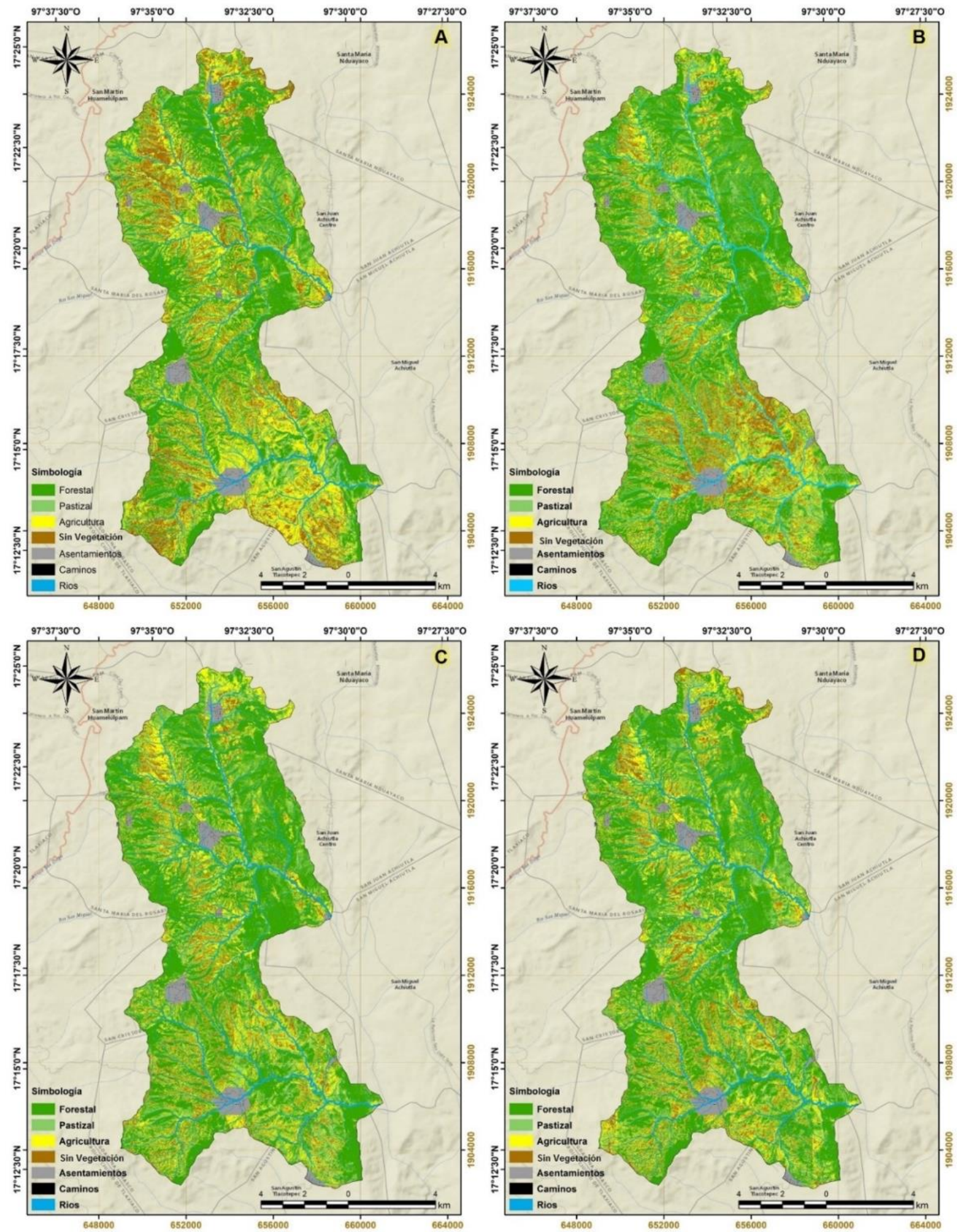

Figura 3. Clasificación del uso de suelo y vegetación durante diferentes años de la microcuenca San Isidro Peñasco, Mixteca Alta, Oaxaca; A) 1995; B) 2010; C) 2013; y D) 2016. 
En 2010, la extensión de la cobertura forestal, en relación con el año 1995 aumentó a $40.5 \%$ (Figura 3B), como consecuencia de las reforestaciones anuales que realizaron diversas comunidades: Ignacio Zaragoza, San Isidro Peñasco, Guadalupe Peñasco, San José del Río, Mirasol y Cavacúa; las cuales, con recursos propios, han plantado más de 600 ha.

A partir del año 1996, las comunidades comenzaron a recibir financiamiento por parte de la Secretaría del Medio Ambiente y Recursos Naturales (Semarnat) por conducto del Programa Nacional de Reforestación (Pronare), posteriormente a cargo de la Comisión Nacional Forestal (Conafor) mediante el Programa de Conservación y Restauración de Ecosistemas Forestales (Procoref), el Programa de Desarrollo Forestal, el Programa ProÁrbol y el Programa Nacional Forestal (Pronafor) para continuar con sus actividades de reforestación; así como para la construcción de viveros y obras de conservación del suelo (Conafor, 2004). Y desde 2019, con el programa Sembrando Vida mediante la implementación de los sistemas de Milpa Intercalada de Árboles Frutales (MIAF) y Sistemas Agroforestales (SAF) para autosuficiencia alimentaria de las familias campesinas (Sader, 2020).

En la clasificación de uso del suelo y vegetación en 2013 y 2016, se observó un cambio mínimo en la cubierta forestal, con respecto al periodo 2010, con una cobertura de $40.6 \%$ y $40.8 \%$ en cada periodo (Figura 3C y $3 \mathrm{D}$ ); debido a las bajas tasas de supervivencia de las reforestaciones y la mortalidad de las especies establecidas en la región; tal es el caso de Pinus greggii Engelm. ex Parl. que logró aclimatarse, pero con el paso de los años murió gradualmente, sin manifestarse su adaptación con la presencia de regeneración natural que permita un aumento en la cobertura forestal (Castellanos-Bolaños y Ruiz, 1993; Velázquez et al., 2003; Valencia et al., 2006; Velasco-Velasco et al., 2012). Con relación al pastizal durante este periodo se advierte un incremento de entre $22.9 \%$ y $25.1 \%$.

Con respecto a las áreas sin vegetación y con erosión, se observó una disminución gradual como resultado del esfuerzo de las comunidades indígenas e instituciones como la Secretaría de Medio Ambiente y Recursos Naturales (Semarnat), la Comisión 
Nacional Forestal (Conafor), la Comisión Nacional de Zonas Áridas (Conaza), el Programa de Naciones Unidas para el Medio Ambiente (PNUMA), Comisión Nacional de Áreas Naturales Protegidas (Conanp), Comisión Nacional del Agua (CNA), Instituto Nacional de Ecología (INE), Comisión Nacional para el Desarrollo de los Pueblos Indígenas (CDI) y Fondo Mundial para la Naturaleza (WWF, por sus siglas en inglés).

\section{Detección de cambio de uso del suelo}

Con las clasificaciones se obtuvieron tres matrices de transición para determinar la pérdida o ganancia, así como una matriz de tasa de cambio. En el periodo 1995-2010, se verificó un incremento de $15.73 \%$ en la cobertura forestal con respecto al año 1995, que se recuperó, principalmente, en áreas de pastizal y agricultura. Al mismo tiempo, el pastizal aumentó a $8.97 \%$; mientras que, en la agricultura y las áreas sin vegetación se registró una reducción de $34.91 \%$ y $18.13 \%$, respectivamente (Cuadro 3 ).

Cuadro 3. Matriz de transición de la cobertura vegetal en los periodos de 1995 al 2010, 2010 al 2013 y 2013 al 2016 (valores expresados en hectáreas).

\begin{tabular}{cccccc}
\hline Año 1995 & Forestal & Pastizal & Agricultura & Sin vegetación & Total \\
\hline Forestal & 5649 & 53 & 25 & 6 & 5734 \\
Pastizal & 679 & 3129 & -957 & 378 & 3229 \\
Agricultura & 445 & 303 & 3743 & -555 & 3936 \\
Sin vegetación & 30 & 32 & 106 & 2049 & 2217 \\
\hline Año 2010 & $\mathbf{6 8 0 4}$ & $\mathbf{3 5 1 8}$ & $\mathbf{2 9 1 8}$ & $\mathbf{1 ~ 8 7 7}$ & $\mathbf{1 5 ~ 1 8 8}$ \\
\hline Forestal & 6656 & 63 & 52 & 33 & 6804 \\
Pastizal & 67 & 3366 & 22 & 63 & 3518 \\
Agricultura & 45 & 368 & 2775 & -270 & 2918 \\
Sin vegetación & 65 & 52 & 11 & 1749 & 1877 \\
\hline Año 2013 & $\mathbf{6 8 3 3}$ & $\mathbf{3 8 4 9}$ & $\mathbf{2 8 6 0}$ & $\mathbf{1 ~ 5 7 5}$ & $\mathbf{1 5 1 1 9}$ \\
\hline Forestal & 6701 & 78 & 36 & 18 & 6833 \\
Pastizal & 73 & 3697 & -221 & 301 & 3850 \\
Agricultura & 80 & 328 & 2706 & -254 & 2860 \\
Sin vegetación & 3 & 128 & 5 & 1439 & 1575 \\
\hline Año 2016 & $\mathbf{6 8 5 7}$ & $\mathbf{4 2 3 1}$ & $\mathbf{2 5 2 6}$ & $\mathbf{1 ~ 5 0 4}$ & $\mathbf{1 5 ~ 1 2 0}$ \\
\hline
\end{tabular}


Para el periodo de 2010-2013, se registró una disminución significativa en las áreas sin vegetación, con una tasa de $16 \%$. El cambio promedio anual para estas fue de 100.6 ha. Además, la agricultura perdió su área en 58 ha $(2.1 \%)$, con un cambio promedio anual de 19.3 ha. En ese lapso, el pastizal aumentó en una proporción significativa con un cambio promedio de $110.3 \mathrm{ha}^{-1} \mathrm{año}^{-1}$. En el marco de tiempo anterior, la cobertura forestal aumentó a una tasa de $0.4 \%$, con un incremento promedio de $9.6 \mathrm{ha}^{-1}$ año-1 (Cuadro 3).

El cambio de uso de suelo durante el periodo 2013-2016 mostró una tendencia similar a la matriz anterior con tasas negativas únicamente para las categorías agricultura y áreas sin vegetación. Los escenarios positivos fueron para las áreas forestales y pastizales con ganancias entre $0.3 \%$ y $9 \%$ (Cuadro 3 ).

De los tipos de uso del suelo y vegetación presentes en la microcuenca, la que registró una mayor tasa de cambio fue el pastizal, al incrementarse $1.3 \%$ por año, lo que se explica por el abandono de las áreas agrícolas ante la pérdida de productividad del suelo. La cobertura forestal también presentó tasas altas de cambio anuales que representaron un incremento de $0.8 \%$ por año, principalmente en la parte alta de la microcuenca (Figura 4). 


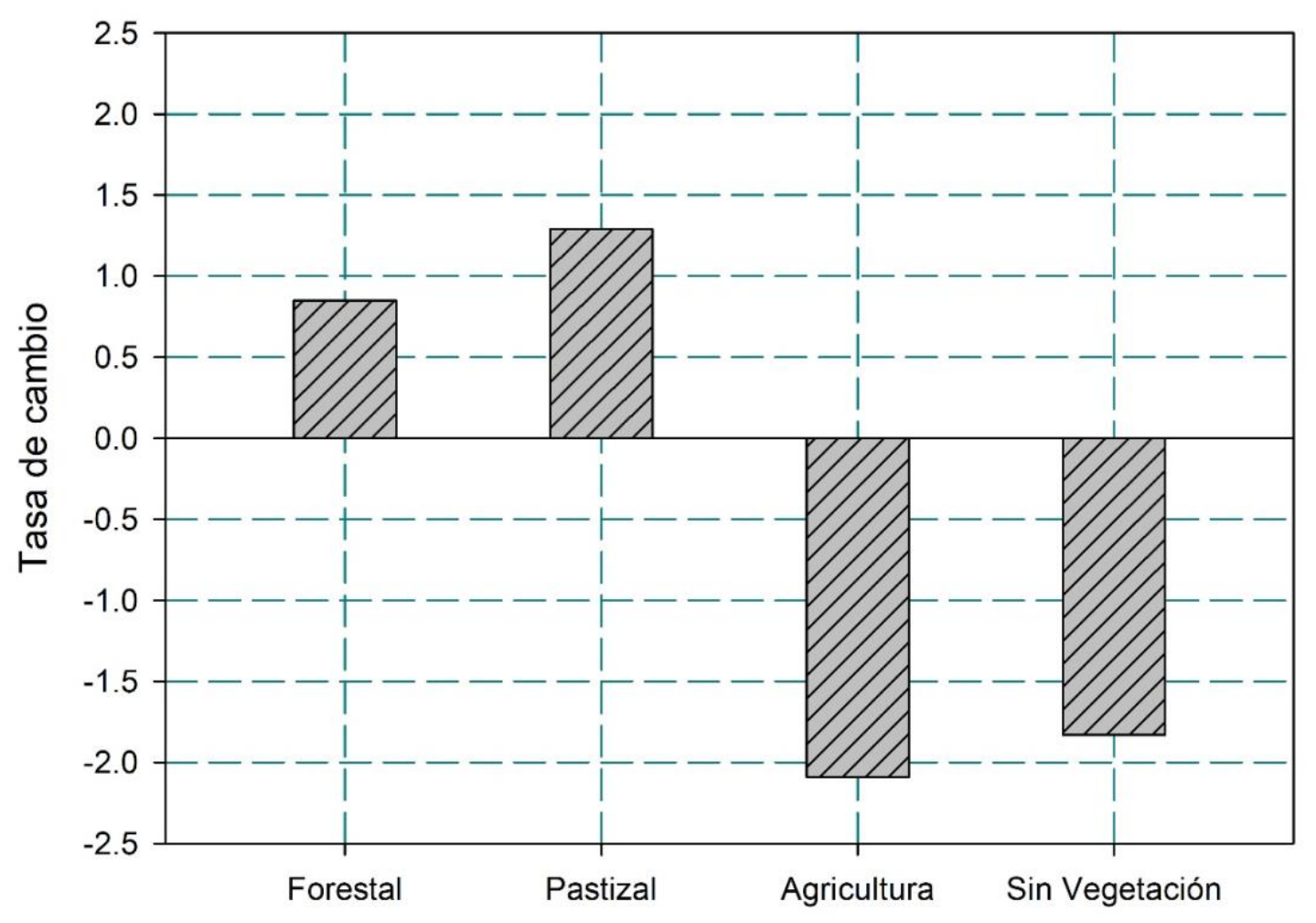

Figura 4. Tasa de cambio de uso del suelo en el área de estudio (1995-2016).

Son diversos los motivos de la dinámica del cambio de uso del suelo y vegetación del área de estudio; entre ellos, el incremento progresivo de la población y las actividades económicas, que propician la expansión de la agricultura, la ganadería, el aprovechamiento selectivo de la madera para la construcción de viviendas; sin dejar a un lado la pérdida de superficie forestal ocasionada por los incendios forestales, así como la presencia de plagas y enfermedades (Contreras-Hinojosa et al., 2003).

La recuperación de la superficie forestal es el producto del esfuerzo realizado por las comunidades que se distribuyen en el área de estudio para reforestar desde la década de los años 90, como parte de una conciencia colectiva para restaurar sus ecosistemas (Carranza, 2014), además de la participación de diversas instituciones que han contribuido significativamente a un manejo integral de la microcuenca para garantizar 
la disponibilidad de bienes y servicios ambientales para las generaciones actuales y futuras (Conafor, 2004).

Con el método de clasificación supervisada de imágenes satelitales de alta resolución, los resultados difieren en gran medida de la información generada por diversas instituciones como la Comisión Nacional para el Conocimiento y Uso de la Biodiversidad (Conabio, 1999), que en comparación con la clasificación del año 1995 del presente estudio presentan una diferencia de $26.2 \%$ para la cobertura forestal y de $63.3 \%$ para la clasificación de agricultura (Figura $5 \mathrm{~A}$ ); lo que se explica por el algoritmo de muestreo utilizado en las imágenes Landsat, cuyo principal insumo es el vecino más próximo que preserva mejor los valores de la radiancia de la imagen; sin embargo, en algunas imágenes se aprecia un desplazamiento entre las coordenadas de las bandas, lo cual puede producir un error sistemático al procesarlas por separado, aunado al error en la captación por parte del sensor de datos, sobre todo en píxeles correspondientes a zonas de traslape con el mismo path, pero diferente row. Estos errores se relacionan con las coordenadas del centro de la imagen utilizado para procesar las imágenes, y para corregirlo se debe realizar un reajuste del origen de la malla de las bandas (Cristóbal et al., 2004). 

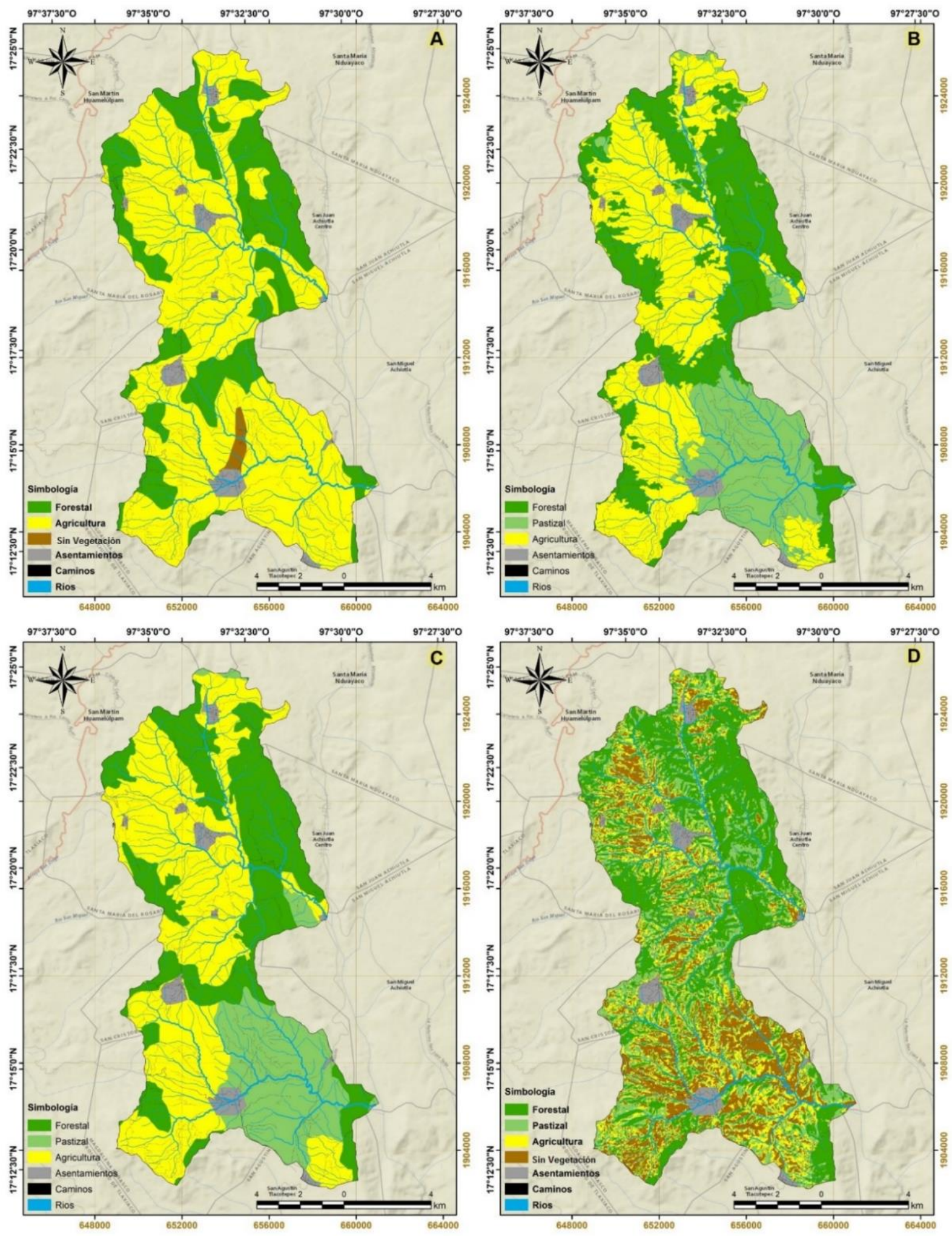

Figura 5. Mapas comparativos de uso de suelo y vegetación en la microcuenca San Isidro Peñasco, Mixteca Alta, Oaxaca; A) Conabio, 1999; B) Conafor, 2014;C) Inegi, 2017; y D) Landsat, 2016. 
La información desarrollada por la Conafor (2013) define una superficie de uso forestal de 5150.2 ha, para pastizal de 3306.4 ha y para agricultura de 6662.3 ha, que comparado con la clasificación del año 2013 supone una diferencia para la cobertura de forestal de $32.7 \%$, pastizal de $16.4 \%$ y agricultura de $57 \%$ (Figura 5B). Respecto a la información generada por Inegi (2017), en la que se establece una superficie forestal de 4852.3 ha, de pastizal de 3117.5 ha y de agricultura con 7149.1 ha, al compararla con la clasificación generada para el año 2016 se obtiene una diferencia para forestal de $41.3 \%$, pastizal de $35.7 \%$ y agricultura de $64.6 \%$ (Figura 5C).

Vega et al. (2008) recomiendan realizar una corrección visual posterior a la clasificación digital de imágenes Landsat, porque la respuesta de la firma espectral varía entre elementos de la misma clase, por el efecto de la sombra provocada por la orografía de la región y la humedad durante la época de adquisición de la imagen; por tal razón, se debe contar con fotografías aéreas analógicas ( $1 \mathrm{~m} /$ pixel), ya que con ellas es posible visualizar con mayor precisión el tipo de uso del suelo y vegetación, lo cual permitirá reducir el margen de error del producto deseado, con una superficie mínima cartografiable de $3 \times 3$ pixeles $(90 \times 90 \mathrm{~m})$.

Con el uso de imágenes satelitales de baja y media resolución disminuye la diferencia de superficie del uso del suelo y la vegetación; por ello, al hacer un análisis comparativo con imágenes tipo Landsat del año 2016 se obtienen coberturas de suelo distribuidas de la siguiente manera: forestal de 5366.6 ha, pastizal con 3733.5 ha, agricultura con 2740.4 ha, áreas sin vegetación 3280.1 ha; y con respecto a las imágenes de alta resolución para ese mismo año, la diferencia para forestal es de $27.7 \%$, pastizal de $13.3 \%$, agricultura de $7.8 \%$ y áreas sin vegetación $54.1 \%$ (Figura 5D).

Los resultados del presente estudio concuerdan con los expresados por Trucíos et al. (2013) en un análisis de la información del Inventario Nacional Forestal (2000), así como la del uso del suelo y vegetación de las series I (1976), II (1992) y III (2002) generada por el Inegi, en comparación con la digitalización e interpretación de ortofotos ( $2 \mathrm{~m} / \mathrm{pixel})$ e imágenes de alta resolución del satélite Quickbird (0.60 
$\mathrm{m} /$ pixel) y Worldview $(0.50 \mathrm{~m} / \mathrm{pixel})$; en el cual se consigna que existen diferencias significativas entre las superficies registradas para forestal, agricultura, pastizal y asentamientos humanos, lo cual se debe a errores de interpretación de imágenes satelitales de 30 y 60 m/pixel durante el Inventario Nacional Forestal y en la información generada por Inegi a una escala 1:250 000; mientras que, dicho error disminuye sustancialmente con el uso de imágenes de alta resolución para una escala de $1: 10000$.

En la investigación que aquí se documenta se indica que existe una mejor clasificación en función de la resolución del pixel, tal como lo demuestra Astola et al. (2019) en un comparativo efectuado entre imágenes satelitales para la predicción de variables forestales en la región boreal; estos autores muestran que hay una mejor capacidad predictiva en los modelos con datos de Sentinel-2 en contraste con Landsat 8, como respuesta a la resolución espacial y las bandas de borde rojo adicionales, lo que permite mejorar la información disponible de los ecosistemas forestales al reducir de 20 a $60 \%$ el sesgo con relación a otros satélites ópticos.

En los últimos 30 años se han hecho trabajos importantes en la microcuenca San Isidro Peñasco de la Mixteca Alta Oaxaqueña para disminuir la presión sobre los ecosistemas, mediante estrategias como: la construcción de presas para retención de sedimentos, zanjas de infiltración, ollas de captación de agua de lluvia, terrazas para estabilización taludes y reforestación con diferentes especies de coníferas. Sin embargo, en la actualidad la microcuenca enfrenta múltiples presiones por las actividades antropogénicas, por lo que se requiere de una priorización de áreas para la restauración ecológica y conservación de la biodiversidad. 


\section{Conclusiones}

En la microcuenca San Isidro Peñasco, la cobertura de los ecosistemas forestales representa $40 \%$ de su superficie, pero es notorio el avance de los pastizales ( $25 \%$ ), la agricultura (15 $\%)$ y de las áreas sin vegetación aparente (9\%). Esta situación permite catalogar a la región como prioritaria para la restauración ecológica, mediante un enfoque de manejo integral del paisaje, debido a la fragmentación de sus ecosistemas.

El uso de las imágenes de alta resolución fundamenta una clasificación de mayor precisión, lo cual garantiza una mejor interpretación de los diferentes escenarios de uso del suelo y vegetación para actualizar la información disponible, lo que permite contar con un panorama real de la superficie de los diferentes tipos de vegetación que requieran estrategias de restauración ecológica y conservación de la biodiversidad a nivel estatal.

\section{Agradecimientos}

Al Consejo Nacional de Ciencia y Tecnología, por las becas de posgrado otorgadas a los dos primeros autores y a la Facultad de Ciencias Forestales de la Universidad Autónoma de Nuevo León.

\section{Conflicto de intereses}

Los autores declaran no tener conflicto de intereses.

\section{Contribución por autor}

Rufino Sandoval-García y Rigoberto González Cubas: diseño, organización, validación y análisis de la información, así como redacción del manuscrito; Javier Jiménez Pérez: revisión y corrección del manuscrito. Todos los autores contribuyeron en la aprobación de la versión final. 


\section{Referencias}

Aguilar-Santelises, R. and R. del Castillo F. 2013. Factors affecting woody plant species diversity of fragmented seasonally dry oak forests in the Mixteca Alta, Oaxaca, Mexico. Revista Mexicana de Biodiversidad 84(2): 575-590. Doi: 10.7550/rmb.30458.

Airbus Defence and Space. 2016. Satellite Maps Yandex. SASPlanet Development Team. http://sat01.maps.yandex.net/tiles?l=sat\&x=(26 de enero de 2020).

Astola H., T. Häme., L. Sirro., M. Molinier and J. Kilpi. 2019. Comparison of Sentinel-2 and Landsat 8 imagery for forest variable prediction in boreal region. Remote Sensing of Environment 223: 257-273. Doi: 10.1016/j.rse.2019.01.019.

Azzari G., M. Jain and D. B. Lobell. 2017. Towards fine resolution global maps of crop yields: Testing multiple methods and satellites in three countries. Remote Sensing of Environment 202: 129-141. Doi: 10.1016/j.rse.2017.04.014.

Birdseye. 2010. Satellite Maps Google. SASPlanet Development Team. http://khms.google.com/kh/v= (26 de enero de 2020).

Caballero, J. J. 2006. Tecnologías tradicionales de transmisión oral en la región de la Mixteca. In: Noriega, M. (ed.). Casa editorial. Manejo del agua y restauración productiva en la región indígena Mixteca de Puebla y Oaxaca. Washington, DC. USA. The international Bank for Reconstruction and Development / The World Bank. México, DF., México. pp. 61-70.

Camacho-Sanabria, J. M., J. I. Juan P., N. B. Pineda J., E. G. Cadena V., L. C. Bravo P. y M. Sánchez L. 2015. Cambios de cobertura/uso del suelo en una porción de la Zona de Transición Mexicana de Montaña. Madera y Bosques 21(1): 93-112. Doi: $10.21829 /$ myb. 2015.211435 . 
Carranza S., J. 2014. Análisis de información espacial y aplicación de herramientas para la determinación de servicios ecosistémicos y toma de decisiones en la Región de la Mixteca. Santa Lucía del Camino, Oaxaca, México. 491 p. http://proyectomixtecasustentableac.org/wp-content/uploads/2018/08/14An\%C3\%A1lisis-de-informaci\%C3\%B3n-espacial-y-aplicaci\%C3\%B3n-deherramientas-de-los-Servicios-Ecosist\%C3\%A9micos.pdf (13 de marzo de 2020).

Castellanos-Bolaños, J. F. y M. Ruiz M. 1993. Introducción de Pinus greggii Engelm. en la Mixteca Alta Oaxaqueña. Folleto de Investigación No. 1. INIF-SARH. Centro de Investigación Pacífico Sur. Yanhuitlán, Oaxaca. México. 18 p. https://vun.inifap.gob.mx/BibliotecaWeb/_Content (17 de mayo de 2020).

Comisión Nacional para el Conocimiento y Uso de la Biodiversidad (Conabio). 1999. Uso de suelo y vegetación modificado por Conabio'. Escala 1: 1000000. Comisión Nacional para el Conocimiento y Uso de la Biodiversidad. Ciudad de México, México. http://www.conabio.gob.mx/informacion/metadata/gis/usv731mgw.xml?_xsl=/db/ metadata/xsl/fgdc_html.xsl\&_indent=no (3 de junio de 2019).

Comisión Nacional Forestal (Conafor). 2004. Manual de obras y prácticas para la conservación de suelos forestales. Ciudad de México, México. 298 p. http://www.conafor.gob.mx:8080/documentos/ver.aspx?articulo $=1310 \& g r u p o=20$ (30 de julio de 2019).

Comisión Nacional Forestal (Conafor). 2013. Inventario Estatal Forestal y de Suelos - Oaxaca. Información complementaria y cartografía. Zapopan Jalisco, México. http://187.218.230.30/filesconafor/userfiles/IEFyS/IEFYS_Oaxaca_2013/ (5 de junio de 2020).

Conrad, O., B. Bechtel, M. Bock, H. Dietrich, E. Fischer, L. Gerlitz, J. Wehberg, V. Wichmann and J. Böhner. 2015. System for Automated Geoscientific Analyses (SAGA) v. 2.1.4. Geoscientific Model Development, 8: 1991-2007.

Doi: 10.5194/gmd-8-1991-2015. 
Coppernicus Open Access Hub. 2020. The european space agencyhe. Missions Sentinel 2A. https://scihub.copernicus.eu/dhus/\#/home (15 de febrero de 2019).

Cristóbal, J., X. Pons y P. Serra. 2004. Sobre el uso operativo de Landsat-7 ETM+ en Europa. Revista de Teledetección 21: 55-59. http://www.aet.org.es/revistas/revista21/AET21-11.pdf (11 de noviembre 2020)

Contreras-Hinojosa, J. R., V. Volke-Haller., J. L. Oropeza-Mota., C. RodríguezFranco., T. Martínez-Saldaña y Á. Martínez-Garza. 2003. Estado actual y causas de la degradación de los agostaderos en el Municipio de Yanhuitlán, Oaxaca. Terra Latinoamericana 21(3): 427-435.

http://www.redalyc.org/pdf/573/57311097001.pdf (25 de marzo de 2020).

Champo-Jiménez, O., L. Valderrama-Landeros y M. L. España-Boquera. 2012. Pérdida de cobertura forestal en la Reserva de la Biósfera Mariposa Monarca, Michoacán, México (2006- 2010). Revista Chapingo Serie Ciencias Forestales y del Ambiente, 18(2): 143-157. Doi: 10.5154/r.rchscfa.2010.09.074.

García, E.-Comisión Nacional para el Conocimiento y Uso de la Biodiversidad (Conabio). 1998. Climas (clasificación de Köppen, modificado por García). Escala 1:1000000. http://www.conabio.gob.mx/informacion/metadata/gis/clima1mgw.xml?_httpcache =yes\&_xsl=/db/metadata/xsl/fgdc_html.xsl\&_indent=no (15 de noviembre 2019).

Guerrero-Arenas, R., E. Jiménez H. y H. Santiago R. 2010. La transformación de los ecosistemas de la Mixteca Alta oaxaqueña desde el Pleistoceno Tardío hasta el Holoceno. Ciencia y Mar 14(40): 61-68. https://biblat.unam.mx/hevila/Cienciaymar/2010/no40/4.pdf (3 de febrero de 2019).

Hastie, T., R. Tibshirani and J. Friedman. 2009. The elements of statistical learning: data mining, inference, and prediction, Springer Series in Statistics. New York, NY. 737 p. Doi: $10.1007 /$ b94608 
Hernández J, E., J. Franco F., A. Villarreal O., C. Camacho J. y M. Pedraza M. 2011. Caracterización socioeconómica y productiva de unidades caprinas familiares en la mixteca poblana. Archivos de Zootecnia, 60(230): 175-182.

Doi: $10.4321 /$ S0004-05922011000200002.

Instituto Nacional de Estadística y Geografía (Inegi). 1976. 'Uso del suelo y vegetación, escala 1:250000, serie I (continuo nacional)', escala: 1:250 000 . Instituto Nacional de Ecología - Dirección de Ordenamiento Ecológico General e Instituto Nacional de Estadística, Geografía e Informática. Digitalización de las cartas de uso del suelo y vegetación elaboradas por Inegi entre los años 1980-1991 con base en fotografías aéreas de 1968-1986.

http://www.conabio.gob.mx/informacion/metadata/gis/usv250kcs1agw.xml?_httpca che=yes\&_xsl=/db/metadata/xsl/fgdc_html.xsl\&_indent=no (15 de enero de 2020).

Instituto Nacional de Estadística y Geografía (Inegi). 1992. 'Uso del suelo y vegetación, escala 1:250000, serie II (continuo nacional)', escala: 1:250 000 . http://www.conabio.gob.mx/informacion/metadata/gis/usv250ks2gw.xml?_httpcach e=yes\&_xsl=/db/metadata/xsl/fgdc_html.xsl\&_indent=no (15 de enero de 2020).

Instituto Nacional de Estadística y Geografía (Inegi). 1995. Conjunto de datos vectoriales. Espacios y Datos de México INEGI. Aguascalientes, Ags., México. https://www.inegi.org.mx/datos/(15 de enero de 2020).

Instituto Nacional de Estadística y Geografía (Inegi). 2002. 'Uso del suelo y vegetación, escala 1:250000, serie II (continuo nacional)', escala: 1:250 000 . http://www.conabio.gob.mx/informacion/metadata/gis/usv250ks3gw.xml?_httpcach e=yes\&_xsl=/db/metadata/xsl/fgdc_html.xsl\&_indent=no (15 de enero de 2020). 
Instituto Nacional de Estadística y Geografía (Inegi). 2004. Síntesis de la información geográfica del estado de Oaxaca. Inegi. Aguascalientes, Ags., México. 180 p. http://internet.contenidos.inegi.org.mx/contenidos/productos/prod_serv/contenidos /espanol/bvinegi/productos/historicos/2104/702825224394/702825224394_1.pdf (3 de enero de 2020).

Instituto Nacional de Estadística y Geografía (Inegi). 2017. Conjunto de datos vectoriales de Uso de Suelo y Vegetación. Escala 1:250 000. Serie VI. Escala: 1:250 000. edición: 1. INEGI. Aguascalientes, Ags., México.

https://www.inegi.org.mx/app/biblioteca/ficha.html?upc $=889463598459$ (12 de marzo de 2019).

Inventario Nacional Forestal. 2000. Sistema Nacional de Información y Gestión Forestal. CONAFOR. https://snigf.cnf.gob.mx/ (15 de febrero de 2020).

Jumb, V., M. Sohani and A. Shrivas. 2014. Color image segmentation using Kmeans clustering and Otsu's adaptive thresholding. International Journal of Innovative Technology and Exploring Engineering 3(9): 72-76. http://www.ijitee.org/wp-content/uploads/papers/v3i9/I1495023914.pdf (3 de febrero de 2019).

Lloyd, S. 1982. Least squares quantization in PCM. IEEE Transactions on Information Theory 28(2): 129-137. Doi: 10.1109/TIT.1982.1056489.

Mendoza G., E. 2002. El ganado comunal en la Mixteca Alta: de la época colonial al siglo XX. El caso de Tepelmeme. Historia Mexicana 51(4): 749-785. https://historiamexicana.colmex.mx/index.php/RHM/article/view/1462/1310 (10 de abril de 2018).

Oliver, A., X. Munoz., J. Batlle., L. Pacheco and J. Freixenet. 2006. Improving clustering algorithms for image segmentation using contour and region information. In: Proceedings of the IEEE International Conference on Automation, Quality and Testing, Robotics 2: 315-320. Doi: 10.1109/AQTR.2006.254652. 
Palacio-Prieto, J. L., M. T. Sánchez-Salazar, J. M. Casado I., E. Propin F., J. Delgado C., A. Velázquez M., L. Chias B., M. I. Ortiz Á., J. González S., G. Negrete F., J. Gabriel M. y R. Márquez H. 2004. Indicadores para la caracterización y ordenamiento del territorio. Instituto Nacional de Ecología-Semarnat, Instituto de Geografía de la UNAM y Secretaría de Desarrollo Social. Convenio Sedesol-Instituto de Geografía, UNAM. México, D. F., México. 161 p.

http://cambioclimatico.gob.mx:8080/xmlui/bitstream/handle/publicaciones/174/43 4_2004_Indicadores_caracter_orden_territorio.pdf?sequence $=1 \& i s A l l o w e d=y$ (26 de febrero de 2020).

Pham, L. T., L. Brabyn and S. Ashraf. 2016. Combining QuickBird, LiDAR, and GIS topography indices to identify a single native tree species in a complex landscape using an object-based classification approach. International Journal of Applied Earth Observation and Geoinformation 50: 187-197. Doi: 10.1016/j.jag.2016.03.015.

QGIS Development Team. 2020. QGIS Geographic Information System. Open Source Geospatial Foundation Project. https://qgis.org (12 de enero de 2020)

Rashmi, C., S. Chaluvaiah and G. H. Kumar. 2016. An efficient parallel block processing approach for K-means algorithm for high resolution orthoimagery satellite images. Procedia Computer Science 89: 623-631. Doi: 10.1016/j.procs.2016.06.025.

Santiago-Mejía, B. E., M. R. Martínez-Menez, E. Rubio-Granados, H. Vaquera-Huerta y J. Sánchez-Escudero. 2018. Variabilidad espacial de propiedades físicas y químicas del suelo en un sistema lama-bordo en la Mixteca Alta de Oaxaca, México. Agricultura, Sociedad y Desarrollo 15(2): 275-288. http://www.scielo.org.mx/pdf/asd/v15n2/1870-5472-asd-15-02-275-en.pdf (26 de febrero de 2019)

SASPlanet, 2020. SASPlanet Software. Versión 181221. SASPlanet Development Team. https://www.sasgis.org (26 de enero de 2020). 
Secretaría de Agricultura y Desarrollo Rural (Sader). 2020. Programa Sembrando Vida. https://www.gob.mx/bienestar/acciones-y-programas/programa-sembrandovida (8 de noviembre 2020).

Servicio Meteorológico Nacional (SMN). 2010. Normales climatológicas, estación 20102 San Agustina Tlacotepec, periodo 1951-2010.

https://smn.conagua.gob.mx/tools/RESOURCES/Normales5110/NORMAL20102.TXT (12 de enero 2019)

Singh, A. 1989. Review article digital change detection techniques using remotelysensed data. International journal of remote sensing 10(6): 989-1003.

Doi: $10.1080 / 01431168908903939$.

Steinhausen M. J., P. D. Wagnerc., B. Narasimhand and B. Waskea. 2018. Combining Sentinel-1 and Sentinel-2 data for improved land use and land cover mapping of monsoon regions. International Journal of Applied Earth Observation and Geoinformation 73: 595-604. Doi: 10.1016/j.jag.2018.08.011.

Trucíos C, R., M. Rivera G., G. Delgado R., J. E. Ávalos y J. Cerano P. 2013. Análisis sobre cambio de uso de suelo en dos escalas de trabajo. Terra Latinoamericana, 31: 339-346. http://www.scielo.org.mx/scielo.php?script=sci_arttext\&pid=S0187$57792013000500339 \&$ Ing $=$ es\&nrm =iso (11 de noviembre 2020)

US Geological Survey (USGS). 2020. Landsat-8 and Sentinel 2A image the Earth Explorer. https://earthexplorer.usgs.gov/ (15 de febrero de 2019).

Valencia M., S., M. V. Velasco G., M. Gómez C., M. Ruiz M. y M. Á. Capó A. 2006. Ensayo de procedencias de Pinus greggii Engelm. en dos localidades de la Mixteca Alta de Oaxaca, México. Revista Fitotecnia Mexicana 29 (1). http://www.redalyc.org/articulo.oa?id=61029104 (15 de febrero de 2020). 
Vega G, A., J. López-García y L. de L. Manzo Delgado. 2008. Análisis espectral y visual de vegetación y uso del suelo con imágenes Landsat ETM+ con apoyo de fotografías aéreas digitales en el Corredor Biológico Chichinautzin, Morelos, México. Investigaciones Geográficas, Boletín del Instituto de Geografía, UNAM 67: 59-75. Doi:10.14350/rig.17990.

Velasco-Velasco, V., J. Enríquez-del Valle, G. Rodríguez-Ortiz, G. Campos-Ángeles, M. Gómez-Cárdenas y M. García-García. 2012. Evaluación de procedencias de Pinus greggii Engelm. Ex Parl. en plantaciones de la Mixteca Oaxaqueña. Revista Mexicana de Ciencias Forestales, 3(9):41-50. Doi: 10.29298/rmcf.v3i9.534.

Velázquez, A., E. Durán., I. Ramírez., J. F. Mas, G. Bocco, G. Ramírez and J. L Palacio. 2003. Land use-cover change processes in highly biodiverse areas: the case of Oaxaca, Mexico. Global Environmental Change 13: 175-184. Doi:

10.1016/S0959-3780(03)00035-9.

World Imagery. 2013. ArcGIS. Imagery. Maps/ESRI, SASPlanet Development Team. https:// www.arcgis.com (26 de enero de 2020).

Todos los textos publicados por la Revista Mexicana de Ciencias Forestales -sin excepciónse distribuyen amparados bajo la licencia Creative Commons 4.0 Atribución-No Comercial (CC BYNC 4.0 Internacional), que permite a terceros utilizar lo publicado siempre que mencionen la autoría del trabajo y a la primera publicación en esta revista. 